\title{
Real Time Tomographic Reflection
}

\author{
Vikram S. Chib \\ Northwestern University, Chicago, Illinois, U.S.A. \\ George D. Stetten \\ University of Pittsburgh, Pittsburgh, Pennsylvania, U.S.A.
}

\section{INTRODUCTION}

Medical imaging modalities are important clinical and diagnostic tools in medicine. Ultrasound, computerized tomography $(\mathrm{CT})$, and magnetic resonance imaging (MRI) provide images that can be used to localize structure and function in the human body; thus, they provide physicians the ability to determine patient abnormalities. However, conventional methods of displaying these imaging modalities are limited since they only allow for viewing images independent from the patient.

\section{OVERVIEW}

While this may not be an issue during non-invasive diagnostic imaging, it becomes one when these images are being used for guidance of surgical procedures. During these situations, the doctor is not able to use direct handeye coordination. Instead, the doctor must look at a display without obvious relation to the position and orientation of his surgical instruments in the operating field. This leads to difficulty in the surgeon's ability to utilize medical image guidance.

In order to allow surgeons a more natural feel during image-guided surgical procedures, there has been a recent push to develop augmented reality systems that seamlessly merge medical images with the direct vision of the surgeon. It is hoped that such systems will enable doctors to employ a more direct form of hand-eye coordination. In essence, medical augmented reality strives to give the surgeon a sort of X-ray vision in order to see directly inside the patient.

While many forms of augmented reality require a headmounted or tracking apparatus, this article describes a particularly simple method called Real Time Tomographic Reflection (RTTR). A specific implementation of RTTR is called the sonic flashlight ${ }^{\mathrm{TM}}$. The sonic flashlight can project a virtual image of an ultrasound scan into its proper visual location within the patient and does not require any tracking or head-mounted apparatus. This system permits the operator to guide a needle through the skin by aiming directly at the ultrasound image and using natural hand-eye coordination rather than looking away from the patient at a screen. It provides an intuitive merger of the visual exterior of the patient with the ultrasound image in situ, which can be shared by others standing next to the operator. The sonic flashlight may increase accuracy, safety, and speed for a wide variety of diagnostic and invasive procedures and enable them to be performed by an operator with less training.

\section{REAL TIME TOMOGRAPHIC REFLECTION}

\section{Augmented Reality and Head-Mounted Displays}

The main approach to fusing images with a direct view of the patient derives from research in augmented reality, so named to differentiate it from the more widely known virtual reality. After all, there is nothing virtual about real image data from real patients. Fuchs, Sauer, and others have developed augmented reality systems based on a head-mounted display (HMD), a type of device that generally replaces direct human vision with miniature video cameras and display screens mounted in front of each eye. ${ }^{[1-5]}$ The HMD permits the merger of a video view of the patient with image data, such as an ultrasound scan, that is rendered from an appropriate perspective. That perspective is determined by tracking both the HMD and the ultrasound transducer, which is generally held by the wearer of the HMD. Primarily because of their complexity, these systems have not been widely accepted. Significant problems remain unresolved, such as limited peripheral vision, resolution lower than human vision, and latency in the image registration. The weight, isolation, and need for a tether have also discouraged clinical use. Some of these problems will undoubtedly be addressed in time, but the HMD system remains inherently a complex and challenging approach. 


\section{Image Overlay}

To reduce the apparatus that the operator must wear, DiGioia and his group at the Carnegie Mellon Robotics Institute have developed a system called image overlay. ${ }^{[6,7]}$ They place the patient beneath a large, halfsilvered mirror. Above the mirror, a flat panel monitor is mounted displaying 3-D renderings of computerized tomography (CT) data. The operator looks down through the mirror at the patient and sees the reflected CT rendering superimposed on the patient. The operator wears only a small, head-tracking optical transmitter, which is required to determine the proper perspective from which the CT data must be rendered for his/her particular viewpoint. A second tracking device must be attached to the patient in order to achieve proper registration between the patient and the CT data that was previously acquired. Special liquid crystal display (LCD) shutter glasses are needed if stereoscopic visualization is desired.

\section{Real Time Tomographic Reflection (RTTR)}

Real Time Tomographic Reflection (RTTR) represents a further simplification to DiGioia's image overlay. With restriction to a single tomographic slice (e.g., ultrasound) and by strategically positioning the transducer, halfsilvered mirror, and display, RTTR eliminates the need for tracking the operator. This is possible because RTTR produces a virtual image. The word virtual is used here in its classical sense: the reflected image is optically indistinguishable from light-emitting voxels suspended in space. A virtual image is stable and independent of viewer location. Furthermore, it produces natural stereoscopic cues without requiring LCD shutter glasses. The operator does not wear any special apparatus, and several viewers can experience the same illusion simultaneously.

This approach is shown in Fig. 1. An ultrasound transducer acquires a tomographic slice representing a set of 3-D voxels in a plane. The image of that slice, which is displayed at its correct size on a flat-panel display, is reflected to occupy the same physical space within the patient. The patient is viewed through the half-silvered mirror. The reflected image is correctly located and independent of viewer location. Since the ultrasound transducer is an integral part of the apparatus, the merger is independent of patient location as well. The image will change during an invasive procedure in order to show the results of the procedure. ${ }^{[8-14]}$

Masamune et al. have used a similar approach, which they call slice display, on CT data. ${ }^{[15]}$ In their first apparatus, the CT scanner was not an integral part; thus, independent registration of patient location was still required. They have since produced a real-time version in which the mirror and monitor are located within the CT scanner. ${ }^{[16]}$ Similar systems have been proposed previ-

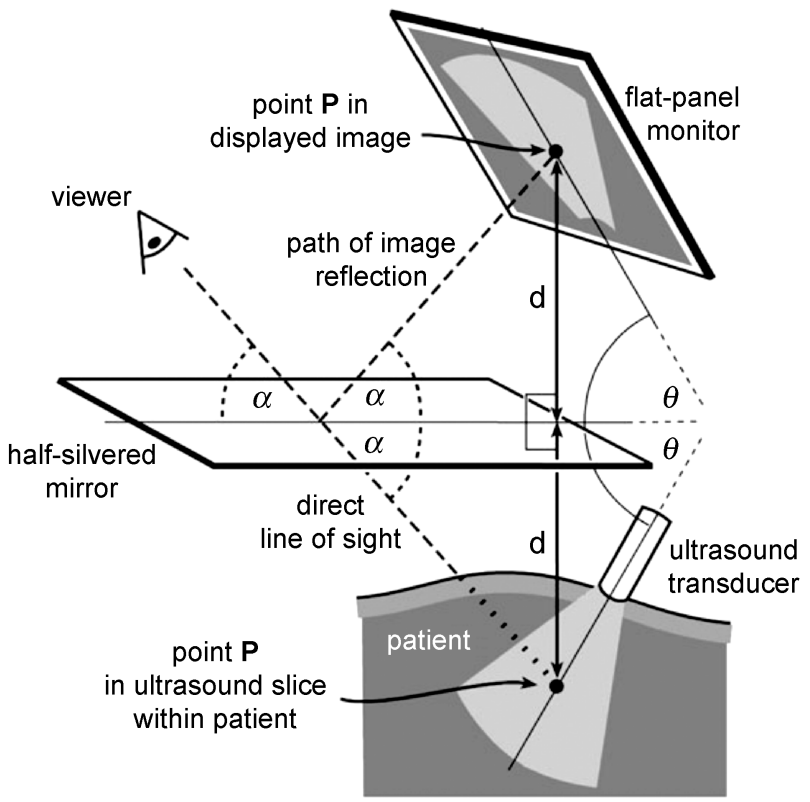

Fig. 1 Geometric relationships for Real Time Tomographic Reflection (RTTR). The mirror must bisect the angle between the slice and the monitor. On the monitor, the image must be correctly scaled, translated, and rotated so that each point in the image is paired with its corresponding point in the slice to define a line segment perpendicular to and bisected by the mirror. By fundamental laws of optics, the ultrasound image will appear at its physical location-independent of viewer position.

ously, though, not for image-guided intervention, including an ultrasound-based system by Hofstein. ${ }^{[17]}$

Figure 2 shows an implementation of RTTR in which a human hand is seen with an ultrasound transducer pressed against the soft tissue between the thumb and index finger. While not a common target for clinical ultrasound, the hand was chosen for this example because it clearly demonstrates successful alignment. The external surfaces of the hand are consistently located with structures in the ultrasound image. The photograph cannot convey the strong sense, which is derived from stereoscopic vision, that the reflected image is located within the hand. This sense is intensified by head motion because the image remains properly aligned from different viewpoints. To one experiencing the technique in person, ultrasound targets within the hand are clearly accessible to direct percutaneous injection, biopsy, or excision. The device was named the sonic flashlight because the transducer appears to illuminate the interior of the hand with the ultrasound data. A U.S. Patent (6599247) on RTTR was issued in 2004.

\section{Geometric transform for calibration}

Clearly, an accurate method for calibration is required. Without it, the sonic flashlight would be unable to guide 
interventional procedures. ${ }^{[14]}$ Calibration requires careful consideration of the degrees of freedom in the registration process. The challenge is to make each pixel in the virtual image occupy and, therefore, appear to emanate from its actual 3-D location in the slice. We first only consider the geometric transform of a rigid body, i.e., we assume that the ultrasound slice is displayed without distortion at its correct scale on a perfectly flat monitor. The geometric transform that would be required to superimpose the virtual image onto the slice can be represented as two sets of translations and rotations. Each of which has three degrees of freedom (Fig. 3). The first (two rotations and one translation) allows the flat-panel display to be moved into its correct plane, which makes the virtual image coplanar with the actual ultrasound slice. We can achieve this by physically moving the display, the ultrasound transducer, or the mirror. The second (two translations and one rotation) can be achieved simply by adjusting the image of the ultrasound slice on the flatpanel monitor.

\section{Affine transform for calibration}

The assumption that both the ultrasound slice and the flatpanel monitor are truly flat is a safe one and makes a rigid-

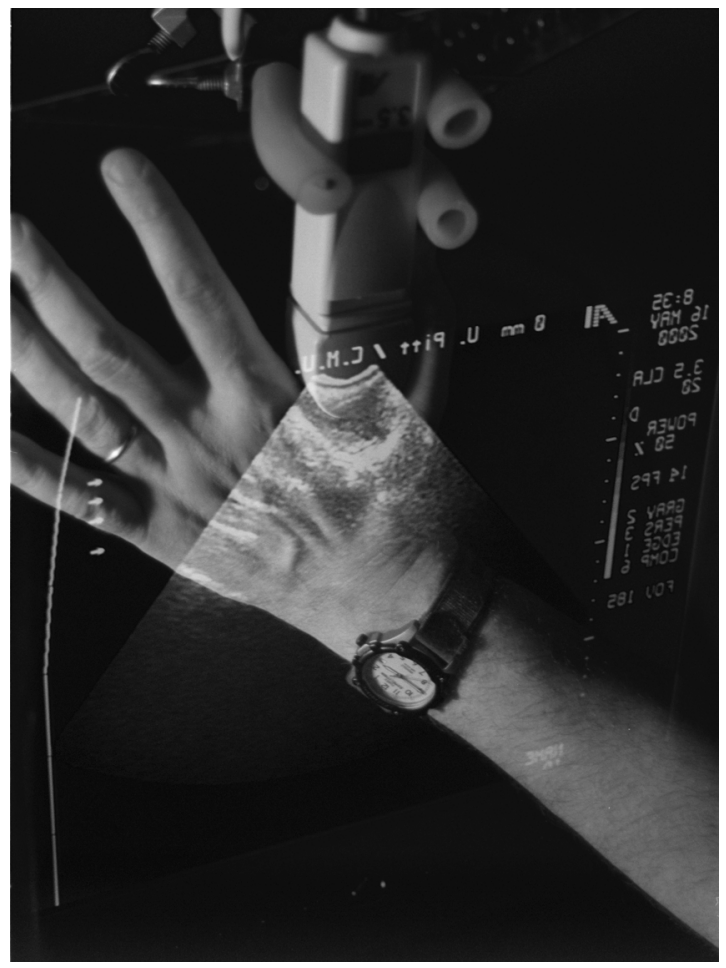

Fig. 2 View of a hand through the half-silvered mirror of the sonic flashlight. (View this art in color at www.dekker.com.)

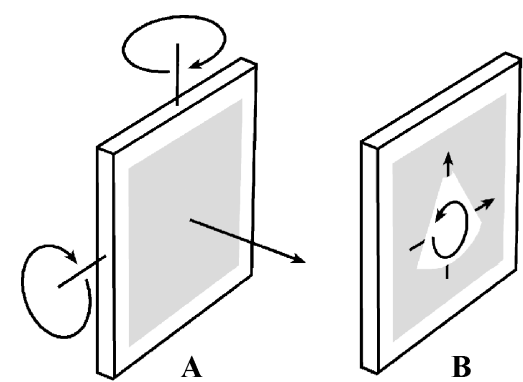

Fig. 3 Geometric transforms for (A) physically moving the display and (B) moving the image on the screen.

body (geometric) transform sufficient to move the monitor into the correct plane. However, correctly adjusting the image on the screen actually requires more degrees of freedom than those provided by the geometric transform-if only because the image must be scaled to its proper size. Extending the geometric transform to an affine transform allows us to scale the ultrasound slice to its correct size, adjust its aspect ratio, and correct for skewing. The calibration process becomes a matter of finding the optimal parameters for that affine transform. Mapping location $(x, y)$ to $\left(x^{\prime}, y^{\prime}\right)$ with an affine transform is accomplished by multiplying the homogeneous form of $(x, y)$ by a $3 \times 3$ matrix $\mathbf{A}$.

$$
\left[\begin{array}{ccc}
x_{1}^{\prime} & x_{2}^{\prime} & x_{3}^{\prime} \\
y_{1}^{\prime} & y_{2}^{\prime} & y_{3}^{\prime} \\
1 & 1 & 1
\end{array}\right]=\left[\begin{array}{ccc}
a_{1,1} & a_{1,2} & a_{1,3} \\
a_{2,1} & a_{2,2} & a_{2,3} \\
0 & 0 & 1
\end{array}\right]\left[\begin{array}{ccc}
x_{1} & x_{2} & x_{3} \\
y_{1} & y_{2} & y_{3} \\
1 & 1 & 1
\end{array}\right]
$$

An affine transform is capable of mapping any triangle onto any other triangle. If the apices of both triangles are known (and not colinear), we can find an explicit solution for the six unknown elements of the matrix A. Calibration can thus be achieved by visually aligning three point targets with their appearances in the virtual ultrasound image.

\section{CONCLUSION}

Superimposing ultrasound images on human vision using Real Time Tomographic Reflection (RTTR) may improve an operator's ability to find targets while avoiding damage to neighboring structures and while generally facilitating interpretation of tomographic images by relating them spatially to external anatomy. As such, it holds promise for increasing accuracy, ease, and safety during percutaneous biopsy of suspected tumors, amniocentesis, fetal surgery, brain surgery, insertion of catheters, and many other interventional procedures. 


\section{ARTICLES OF FURTHER INTEREST}

3-D Medical Imaging, p. 1

Nuclear Magnetic Resonance Spectroscopy, p. 1084

\section{REFERENCES}

1. State, A.; Livingston, M.; Garret, W.; Hirota, G.; Whitton, M.; Pisano, E.; Fuchs, H. Technologies for Augmented Reality Systems: Realizing Ultrasound-Guided Needle Biopsies. In Computer Graphics Proceedings, Annual Conference Series, Proceedings of SIGGRAPH, New Orleans, LA, 1996; 439-446.

2. Fuchs, H.; State, A.; Pisano, E.; Garret, W.; Hirota, G.; Livingston, M.; Whitton, M.; Pizer, S. Towards Performing Ultrasound-Guided Needle Biopsies from Within a Head-Mounted Display. In Visualization in Biomedical Computing; Springer Verlag: Hamburg, Germany, 1996; 591-600.

3. Fuchs, H.; Livingston, M.; Raskar, R.; Colucci, D.; Keller, K.; State, A. Augmented Reality Visualization for Laparoscopic Surgery. In Lecture Notes in Computer Science, Proceedings of MICCAI, Cambridge, MA, 1998; 934943.

4. Sauer, F.; Khamene, A.; Bascle, B.; Schimmang, L.; Wenzel, F.; Vogt, S. Augmented Reality Visualization of Ultrasound Images: System Description, Calibration, and Features. In IEEE ACM International Symposium on Augmented Reality; IEEE Computer Society: New York, 2001; 30-39.

5. Azuma, R.T. A survey of augmented reality. Teleoperators and virtual environments. 1997, 6 (4), 355-385.

6. DiGioia, A.; Colgan, B.; Koerbel, N. Computer-Aided Surgery. In Cybersurgery; Satava, R., Ed.; Wiley: New York, 1998; 121-139.

7. Blackwell, M.; Morgan, F.; DiGioia, A. Augmented reality and its future in orthopaedics. Clin. Orthop. Relat. Res. 1998, 345, 111-122.
8. Stetten, G.; Chib, V.; Tamburo, R. System for LocationMerging Ultrasound Images with Human Vision. In Applied Imagery Pattern Recognition (AIPR) Workshop; IEEE Computer Society: Washington, DC, 2000; 200-205.

9. Stetten, G.; Chib, V. Real Time Tomographic Reflection with Ultrasound: Stationary and Hand-Held Implementations; Carnegie Mellon University, Nov. 2000. Robotics Institute Technical Report \# CMU-RI-TR-000-28. http:// www.ri.cmu.edu/pubs/pub_3412.html\#text_ref.

10. Stetten, G.; Chib, V. Overlaying ultrasound images on direct vision. J. Ultrasound Med. 2001, 20 (1), 235-240.

11. Stetten, G.; Chib, V. Magnified Real-Time Tomographic Reflection. In Lecture Notes in Computer Science, Proceedings of MICCAI, Springer Verlag, 2001; 683-690.

12. Stetten, G.; Shelton, D.; Chang, W.; Chib, V.; Tamburo, R.; Hildebrand, D.; Lobes, L.; Sumkin, J. Towards a Clinically Useful Sonic Flashlight. In Proceedings of the IEEE International Symposium on Biomedical Imaging; IEEE Computer Society: Washington, DC, 2002.

13. Chang, W.; Stetten, G.; Lobes, L.; Shelton, D.; Tamburo, R. Guidance of retrobulbar injection with real time tomographic reflection. J. Ultrasound Med. 2002, 21, 1131-1135.

14. Stetten, G.; Chib, V.; Hildebrand, D.; Bursee, J. Real Time Tomographic Reflection: Phantoms for Calibration and Biopsy. In IEEE ACM International Symposium on Augmented Reality; IEEE Computer Society: New York, 2001; 11-18.

15. Masamune, K.; Masutani, Y.; Nakajima, S.; Sakuma, I.; Dohi, T.; Iseki, H.; Takakura, K. Three-Dimensional Slice Image Overlay System with Accurate Depth Perception for Surgery. In Lecture Notes in Computer Science, Proceedings of MICCAI, Pittsburgh, PA, 2000; 395-402.

16. Masamune, K.; Fichtinger, G.; Deguet, A.; Matsuka, D.; Taylor, R. An Image Overlay System with Enhanced Reality for Percutaneous Therapy Performed Inside CT Scanner. In Lecture Notes in Computer Science, Proceedings of MICCAI, Springer Verlag, 2002; 77-84.

17. Hofstein, S.R. Ultrasonic Scope. US Patent 956899, April 29, 1980. 